\title{
Instantaneous Penetration Level Limits of Non-Synchronous Devices in the British Power System
}

\author{
Mengran $\mathrm{Yu}^{1 *}$, Andrew J. Roscoe ${ }^{1}$, Adam Dyśko ${ }^{1}$, Campbell D. Booth ${ }^{1}$, Richard Ierna ${ }^{2}$, Jiebei \\ $\mathrm{Zhu}^{2}$, Helge Urdal ${ }^{3}$ \\ ${ }^{1}$ Department of Electronic and Electrical Engineering, University of Strathclyde, Glasgow, \\ United Kingdom \\ ${ }^{2}$ Department of System Operators, National Grid, Warwick, United Kingdom \\ ${ }^{3}$ Urdal Power Solution Ltd., Warwick, United Kingdom \\ *mengran.yu@strath.ac.uk
}

\begin{abstract}
The installed capacity of non-synchronous devices (NSD), including renewable energy generation and other converter-interfaced equipment such as energy storage, bi-directional transfer links, electric vehicles, etc., is expected to increase and contribute a large proportion of total generation capacity in future power systems. Concerns have been expressed relating to operability and stability of systems with high penetrations of NSD, since NSD are typically decoupled from the grid via power electronic devices and consequently reduce the "natural" inertia, short-circuit levels and damping effects which are inherently provided by synchronous machines. It is therefore crucial to ensure secure and stable operation of power systems with high penetrations of NSD.

This paper will show and quantify the instantaneous penetration level (IPL) limits of NSD connected to a simple example power system in terms of steady-state stability beyond which the system can become unstable or unacceptable, defined as "unviable". The NSD used in this example will be a conventional $d q$-axis current injection (DQCI) convertor model. The paper will introduce a set of criteria relating to locking signal in converter phase-locked loop, frequency, rate of change of frequency and voltage magnitude, which will be used to determine the system viability and the IPL limit. It will also be shown that there are several factors that can potentially affect the IPL limits. Frequency and voltage droop slopes and filter time-constant for DQCI converter are varied and it is shown how these settings influence the IPL limits. Finally, to provide additional insight into network viability under high penetrations of NSD, a visualisation method referred here as "network frequency perturbation" is introduced to investigate responses of individual generators to a change in network frequency.
\end{abstract}

\section{Introduction}

The installed capacity of non-synchronous devices (NSD) in power networks including renewable energy sources and other converter-interfaced devices such as high voltage direct current (HVDC) links, energy storage, electric vehicles, etc., is expected to increase significantly in the near future. According to [1, 2] under the 'Gone Green' scenario, the percentage of electrical power produced using renewable energy sources in Great Britain (GB) may increase to a total proportion of 33\% in 2020/21, 48\% in 2030/31, and is expected to increase further in the following years. Over the time period from 2020 to 2030, the installed capacity of interconnectors (to other countries in Europe) is predicted to increase by approximately $6 \mathrm{GW}$ in the 'Gone Green' scenario [1].

In conventional $d q$-axis current injection (DQCI) converters for wind turbines and voltage source converter (VSC) based HVDC links, active and reactive power setpoints are translated to $d q$-axis current $\left(I_{d q}\right)$ 
references in the rotating reference frame. The DQCI control algorithm is a well-established method described in many publications (e.g. [3]). High-bandwidth inner current control loops are used to control the modulated voltage waveforms at the switching bridges or multi-level modules to ensure that the actual injected currents closely match the $I_{d q}$ references. Such converters, therefore, aim to present high impedances from the perspective of unbalance, harmonics and inter-harmonics. The currents output by converter-interfaced sources aim to be close to balanced positive-sequence sinusoids, as required by the present GB and European Grid Codes [4-6], even in the presence of voltage unbalance or harmonics. DQCI controllers are normally designed for connection to strong alternating current (AC) systems, e.g. when short-circuit ratios are higher than 3.0 [7], and operate under the assumption that an infinite bus or a large positive-sequence balanced voltage source, such as a large aggregated capacity of synchronous machines (SM), is present in the network. However, in future power networks, such assumptions may no longer hold. As the proportion of DQCI converter capacity rises, the effective grid impedance between the SM voltage sources and the converters increases. Eventually, a point will be reached where the aggregated transient reactance $\left(X_{d}^{\prime}\right)$ is so large that, when added to the grid impedance, it causes the DQCI converters to become unstable. This effect has been discussed in $[8,9]$. The instability is caused by the high bandwidth voltage disturbances at the connection point (CP), due to the high currents from the aggregated converters, and the large grid impedance. The frequency at which instability occurs could lie anywhere within a wide frequency region, depending on the exact controller parameters, impedances, ratings/capacities, and set-points.

In recent years, the issues raised by high penetrations of NSD have been widely discussed in both industry and in the literature. For example $[9,10]$ discuss interactions of the inner vector current controller with low-frequency resonances, and negative effects of the phase-locked loop (PLL) dynamics applied in the inner current control. Other effects of high penetration of NSD on power network performance, include network frequency stability [11,12], rotor angle stability [13, 14], voltage stability [13, 15], and small signal stability [16]. Studies have also been conducted relating to NSD penetration level limits. For example, [11] shows a maximum limitation of the ratio of the total amount of energy derived from NSD to the overall system rotational energy to be 0.17 in order to maintain system security in terms of rate of change of frequency (RoCoF) criteria. [17] investigates system performance when there is up to a $30 \%$ penetration level of distributed generation resources (DGR), and investigates the effects of changes in location and type of DGR connected. Studies in [18] have shown that the maximum instantaneous penetration level (IPL) for NSD, in terms of angular stability, is in the region of $65 \%$ of dispatched generation or $75 \%$ in terms of connected generation capacity for the GB power system. Work in [18] is based on a power system model using phasor simulation (i.e. not transient analysis). However, to date there have been limited studies conducted with the objective of establishing IPL limits of NSD, i.e. the "tipping points", based on a set of realistic criteria from the network operator perspective, (including locking signal in the PLL of the DQCI converter control algorithm, frequency, RoCoF and voltage magnitude), using high-fidelity time-domain dynamic power system models. In the following sections, it will be shown that there are IPL limits for NSD with conventional DQCI 
control, beyond which the system state becomes unacceptable (termed as "unviable" in the paper). A set of criteria to determine system viability will be introduced and the IPL limits based on such criteria will be assessed.

The IPL limits can be affected by many factors, such as system configuration, overall loading levels, types of generation, converter controller settings, etc. It is important to understand the effects of these factors on IPL limits, so that device types, algorithms and configurations can be chosen to facilitate the required high penetrations of converters in the network. The number of parameters affecting the IPL limit is large, and so finding an "optimum" parameter set and mix of generation/converter types is practically impossible, since it would involve exploration of a multi-dimensional search space with a high number of parameters. However, in this paper, certain key parameters such as frequency and voltage droop slopes and filter time-constant are chosen, and explored in isolation. Then, by selecting the most promising values from each "slice" of the search space, a configuration for DQCI converter settings which appears to offer the highest possible IPL will be selected. It should be noted that the paper does not aim to establish global parameter optimisation, but provides more of an experimental (simulation based) approach. Additionally, a visualisation method for investigating individual generator response to an enforced change in network frequency, termed "network frequency perturbation (NFP)" will also be introduced in this paper. The NFP method is not a classical stability assessment technique such as the state-space analysis used in control system design, but offers a useful way of investigating and visualising generator behaviour in response to network disturbances. It offers an additional insight into potential interactions (e.g. unstable oscillatory modes) between different generating technologies.

The paper is organised as follows. The definitions and the analysis methods are introduced in section 2. Dynamic modelling of the power system and a set of complex criteria to determine system viability are presented in section 3, along with analyses of case studies related to IPL limits. In section 4 the NFP method is introduced, and the frequency responses of SG and DQCI are presented to gain better understanding of the contributions of individual NSDs. The conclusions of the paper and recommendations for further research are presented in section 4 .

\section{Definitions and methodology}

\subsection{Instantaneous Penetration Level (IPL) definition and optimisation of controller parameters}

In this paper, IPL is defined as the ratio of instantaneous power output of the NSD to the total system demand in a power network, as shown in (1). Although various definitions of IPL have been used in technical literature $[18,19]$, the authors of this paper believe that definition (1) presents the best intuitive indication of the percentage of overall generation being supplied from the NSD for a given demand.

$$
I P L=\frac{P_{N S D}}{P_{\text {Demand }}} \times 100 \%
$$


It is important to understand the effects introduced by various parameters and controller settings on the IPL limits, which can support the optimisation of the existing methods as well as the development of new techniques to maximise the NSD penetration in a network. There are many factors that can affect the IPL limit, including controller gains, filter time constants, gains in ramp rate limiters, system impedance (including generation reactance and transmission line impedance), the types and magnitudes of load, etc. The assessment of all possible influencing factors is very complex and goes beyond the limits of a single journal paper. The investigation presented in this paper concentrates on the detailed evaluation of four parameters, namely voltage and frequency controller droop slopes and low-pass filter (LPF) time constants. In order to maximise the IPL limit, a simulation based method is introduced which applies to any two groups of settings. A group of settings in this context is defined as a small set (e.g. two) of parameters/factors for which the optimal (maximum) IPL limit can be found. The basic concept is to select the highest IPL limit that can be achieved independently by each group of settings, and then determine the resulting IPL limit by combining the two individually optimised groups. As the presumption cannot be made that the system is linear, the combined effect of the two optimised groups may not always produce the highest achievable IPL limit and further iterations may be needed to achieve the global IPL maximum.

\subsection{Network Frequency Perturbation (NFP) visualisation method}

In a meshed AC electrical network with many generators and loads, frequency changes continuously. In order to gain an understanding of how individual devices contribute to frequency stability and active power balance management, it is useful to examine how a device responds to a change in network frequency. The NFP method allows a clear distinction to be made between devices that provide frequency support through droop-slope type response, and inertial-type response. The method is not a classical stability assessment technique. However, the results (Bode type plots) give a useful graphical insight into the device behaviour during network disturbances, and may offer indication of potential instability under certain NSD penetration levels.

The NFP method introduced in this paper places the device within a hypothetical or 'test' power system, in which frequency is forced and modulated in a sinusoidal fashion at frequency $f_{N F P m o d}$, with a small amplitude $\Delta f$ (assumed to be $1 \%$ in this paper) about the nominal frequency $f_{0}$. This can be expressed as:

$$
f=f_{0}+\Delta f \cos \left(2 \pi f_{N F P \text { mod }}\right)
$$

The value of $f_{N F P m o d}$ changes from $10^{-3} \mathrm{~Hz}$ to $20 \mathrm{~Hz}$. The device responds to this changing frequency with a modulated active power output:

$$
P_{\text {out }}=P_{\text {set }}+\Delta P \cos \left(2 \pi f_{N F P_{\text {mod }}}+\phi_{\Delta P}\right)
$$

The amplitude of the frequency modulation $\Delta f$ is kept small so that no unnatural saturation of device control loops occur. The response parameters $\Delta P$, where $\Delta P$ is in per-unit (pu), and $\phi_{\Delta P}$ together form a response $R$, when normalised to $\Delta f(4)$. 


$$
R=\frac{\Delta P \angle \phi_{\Delta P}}{\left(\frac{\Delta f}{f_{0}}\right)}
$$

$\Delta \mathrm{P}$ and $\phi_{\Delta P}$ can be found either by classical analysis of the device transfer functions, or by time-domain simulation of the entire device and its controllers followed by Fourier Analysis of the output power $P_{\text {out }}$. In both cases, the amplitude of the voltage is assumed to remain constant at $1 \mathrm{pu}$, so that the analysis is purely an examination of the interaction between active power and frequency. The NFP charts for amplitude and phase are plotted by setting $f_{N F P m o d}$ on the $\mathrm{x}$ axis, and plotting the amplitude and phase of $R$.

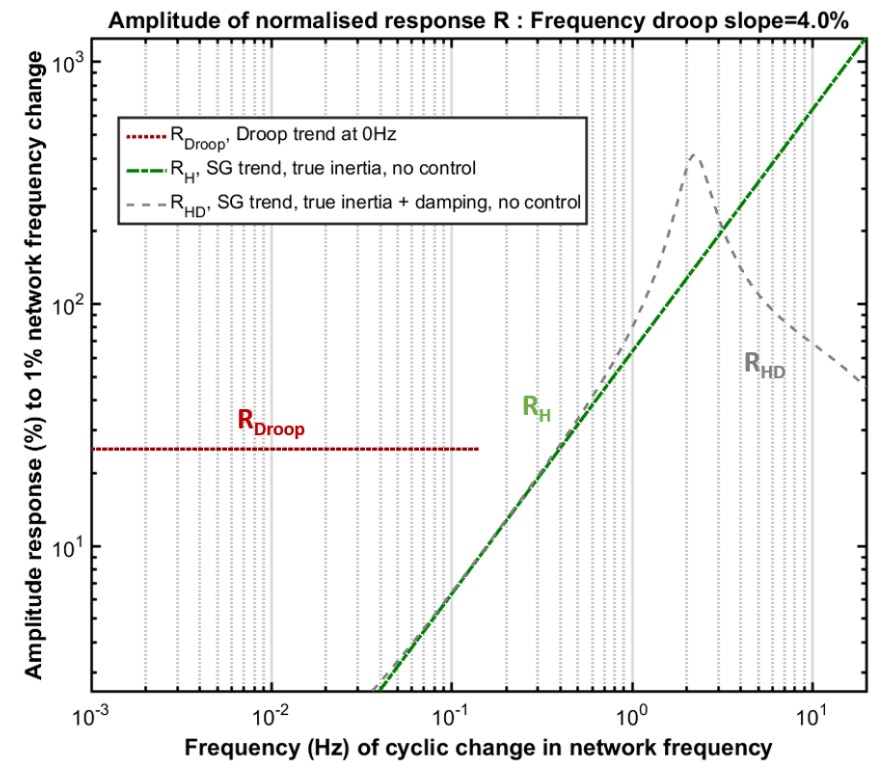

(a) Amplitude chart

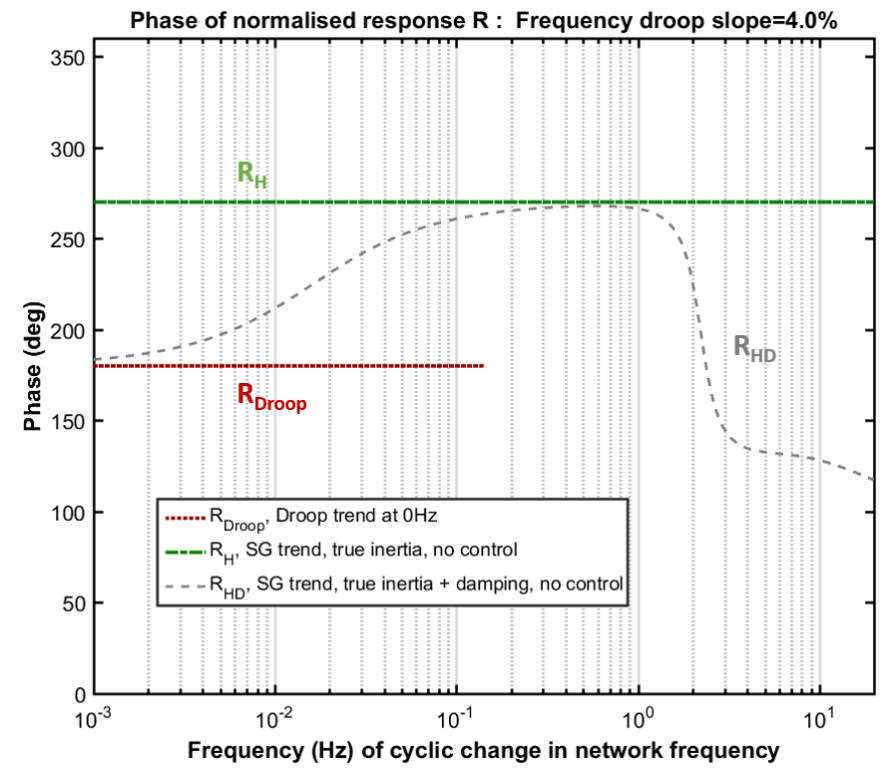

(b) Phase chart

Fig. 1. Illustration of the key featured responses $R$ introduced by the NFP Method based on linear analysis method

On this chart there are three key features, plus a number of more subtle details which can be interpreted to give further understanding of the device response, as shown in Fig. 1 (a) and (b) for amplitude and phase respectively. Note that asymptotes $R_{\text {Droop }}, R_{H}$ and $R_{H D}$ shown in Fig. 1 are obtained from classical linear analysis. Firstly, if the generator is configured with a governor and frequency droop, this creates an asymptote where $R \rightarrow-1 / D_{f}$ as $f_{\text {NFPmod }} \rightarrow 0$ where $D_{f}$ is the frequency droop slope. If the governor and droop slope are operating correctly, then the device response must approach this asymptote, as the frequency of the perturbation tends to zero, which is illustrated as response $R_{\text {Droop }}$. The amplitude of $R_{\text {Droop }}$ is expected to stay at $25 \%$ of $\Delta P$ (due to the assumed $4 \%$ frequency droop slop), and on the phase diagram at $180^{\circ}$, due to the inverse relationship between active power and frequency in the drooped relationship. Secondly, if the generator has a true inertial response, this creates a second key asymptote $R_{H}$ as depicted in Fig. 1 (drawn for the idealistic synchronous machine without damping), and is expressed by equation (5). The response must intercept this asymptote (in both amplitude and the $90^{\circ}$ phase advance relative to the droop asymptote) typically for frequencies between around $0.04 \mathrm{~Hz}$ and $2 \mathrm{~Hz}$, shown as response $R_{H}$. The third key feature is that for any device which intercepts the inertia asymptote $R_{H}$, there must be a resonant peak that occurs, typically in the region of $1-3 \mathrm{~Hz}$, but this is dependent on inertia (real or synthetic), impedance $X_{d}$ ' and 
damping (real or synthetic), shown as response $R_{H D}$. Both responses $R_{H}$ and $R_{H D}$ are presented with the assumption that there is no governor controller attached to the generator (i.e. the machine operates like a synchronous compensator or a flywheel). NFP response plots of SG and DQCI converter will be introduced and analysed in section 3.4.

$$
R_{H}=-j 2 H\left(\frac{f_{N F P_{\text {mod }}}}{f_{0}}\right)
$$

\section{Simulation model and case studies}

\subsection{Power system model}

In order to investigate the IPL limits of NSD, a power system model was built in Matlab SimPowerSystems as shown in Fig. 2(a). The model includes a single/aggregated SM, and a single/aggregated DQCI converter. A network and aggregated resistive load are rated at $10 \mathrm{GW}$. Power setpoints of each type of generation can be varied to support the load in the system and the MVA rating/MW output of each type of generation can be varied to achieve different converter IPLs. The system nominal voltage is $275 \mathrm{kV}$. Although the earlier study [18] utilised a larger multi-generator model it was built with much lower fidelity, i.e. phasor based simulation was used. As one of the key aims of this study is to gain deeper understanding of the key phenomena and interactions at various frequencies which can affect system stability, a higher fidelity generation model was developed for the purposes of this paper, while the simplified model architecture allows for much clearer interpretation of the results. Subsequent studies will include a more comprehensive multigenerator model and /or converter hardware-in-loop (CHIL) environment.

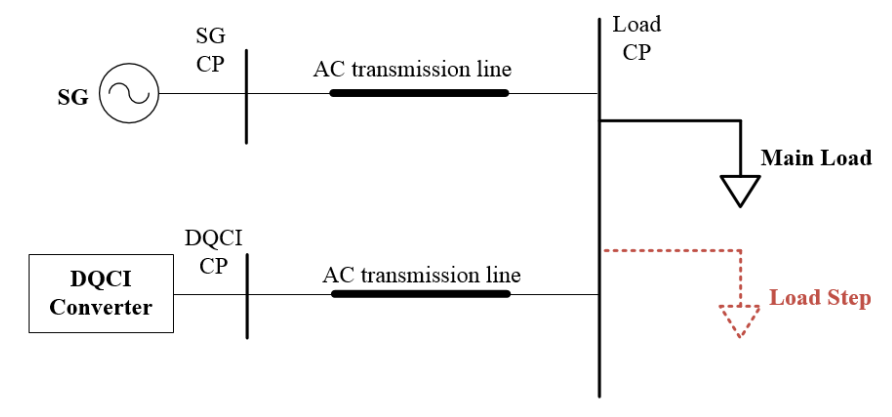

(a) Power system model

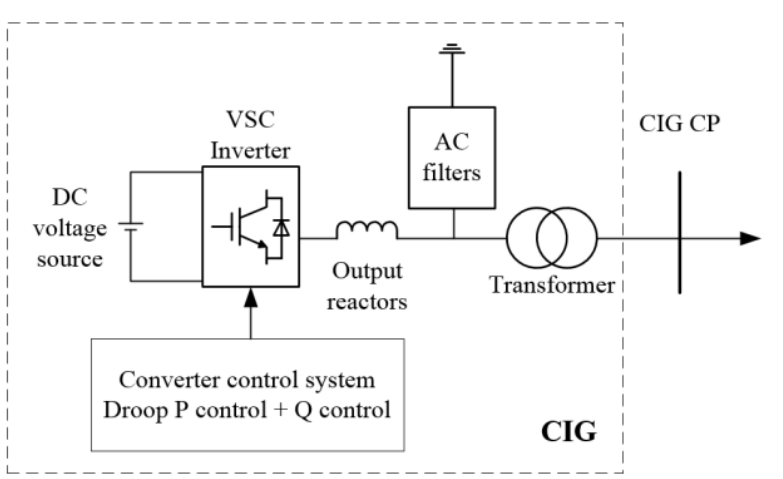

(b) Model of DQCI converter

Fig. 2. Configuration of power system model in Matlab SimPowerSystems

A standard SM model included in the SimPowerSystems library has been used in the model, with standard IEEEG1 steam turbine and governor model [20] and AC1A excitation system applied as the control system for the synchronous generator (SG). The DQCI converter is modelled as the inverter side of a VSCbased HVDC transmission system, which is connected to a DC bus with an assumption of a constant and effectively-controlled DC link voltage, as shown in Fig. 2(b). Conventional active and reactive power control of VSC-HVDC transmission system are implemented for the DQCI converter. Additionally, a frequency droop control is implemented to control the active power setpoint and a voltage droop control is implemented to control the reactive power setpoint, both of which will be required to manage frequency and voltage if these 
converters are used at high penetrations. First-order LPFs are applied at the droop controller outputs separately, i.e. on the sum of power setpoint and power adjustment from the droop controller, to filter out the highfrequency components and limit the converter power ramp-rate. The $d q$-axis current references are also ratelimited to $10 \mathrm{pu} / \mathrm{s}[6,21]$, which helps to manage converter start-up and HVDC link management. Capacity factors of the SG and DQCI converter are set to $60 \%$ and $30 \%$ respectively. AC transmission lines are modelled as series RL circuits. A small $1 \%$ resistive load step (with respect to the main load) is applied for testing system steady-state stability.

In these simulations with high penetrations of converter-connected generation, initialising the model components proved particularly difficult, i.e. machines, governors, automatic voltage regulators (AVRs), and all converter components including PLLs and $d q$-axis control loops. The simulation must not contain an infinite bus since frequency, voltage and power-quality must be allowed to deviate from nominal as this is a crucial part of the simulation. The simulation therefore begins with a carefully orchestrated initialisation process, which lasts 8 seconds, during which the generators and converters are all synchronised, set-points and power flows established at equilibrium, and finally the infinite bus is removed.

\subsection{Criteria for power system viability}

A small disturbance, $1 \%$ resistive load step is then applied to test the system stability. This test event occurs at $15 \mathrm{~s}$, with the simulation running for a further $10 \mathrm{~s}$. If any of the following conditions occur during the simulation the system is considered to be either unstable or have unacceptable performance and is defined as "unviable" in this paper:

- A locking signal from the PLL in the DQCI converter is unlocked for a period longer than $1.5 \mathrm{~s}$, any time after $t=16.5 \mathrm{~s}$, i.e. $1.5 \mathrm{~s}$ after the disturbance (fundamentally, the converter is no longer controlled if the PLL within the converter is unlocked, and the gate switching signals will be turned off to avoid damage to the converter);

- The frequency at the DQCI converter terminal is higher than $52 \mathrm{~Hz}$ or lower than $47 \mathrm{~Hz}$ for a period longer than $500 \mathrm{~ms}$, with reference to [6,22], at any time from $t=10 \mathrm{~s}$ onwards (this is because in some cases, the instability occurs after the infinite bus is removed and the model cannot converge and survive until the load step event);

- The RoCoF at the DQCI converter terminal is higher than $1 \mathrm{~Hz} / \mathrm{s}$, at any time after $t=15.5 \mathrm{~s}$ [22];

- The voltage at the DQCI converter terminal exceeds $\pm 10 \%$ of the nominal voltage level, at any time after $t=16.5 \mathrm{~s}[4,6]$.

\subsection{Effects of frequency and voltage droop controllers on the IPL limits}

Four of the many parameters selected, which have the potential to affect the IPL limit of DQCI convertor are, the droop slopes $\left(D_{f}\right.$ and $\left.D_{v}\right)$ and time constants $\left(\tau_{L P F_{-} D f}\right.$ and $\left.\tau_{L P F_{-} D v}\right)$ in the LPFs of both the frequency and voltage droop controllers. Their effects on the IPL limits are shown in Fig. 3 (a) and (b) for frequency and voltage droop controllers respectively. For both controllers, the droop slope range is selected from $4 \%$ (an aggressive droop response) to infinite (the actual setting in the model is $1 e^{9}$ ) representing no frequency or 
voltage droop from the DQCI converter. The time constants in the LPFs taken are varied from $0.04 \mathrm{~s}$ to $0.4 \mathrm{~s}$. Note that when studying the effects of frequency droop controller, the voltage droop controller is disabled by setting the droop slope $D_{v}$ to infinite and vice versa.

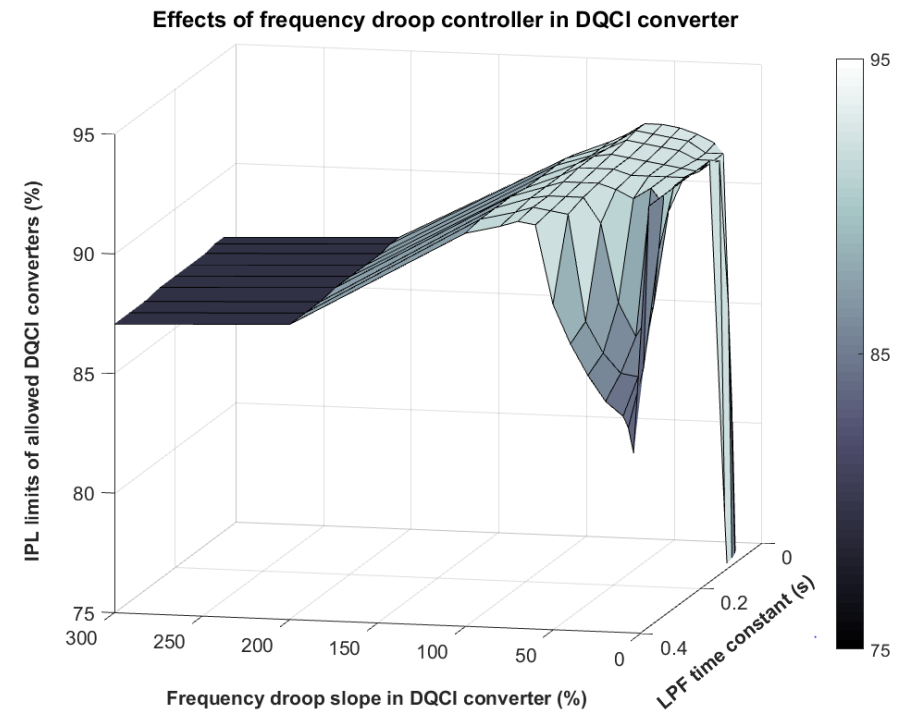

(a) Effects of frequency droop controller

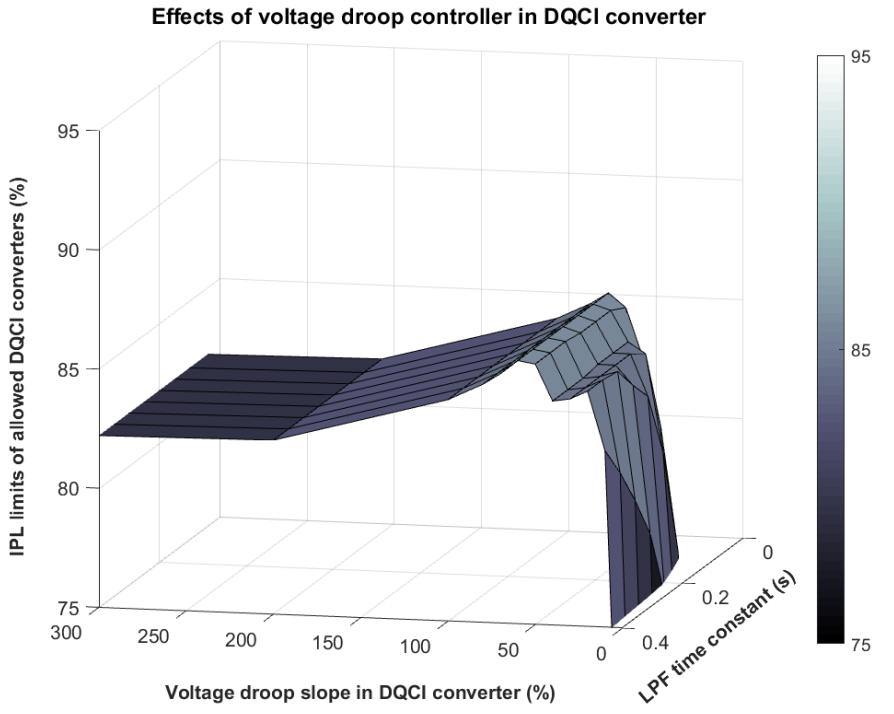

(b) Effects of voltage droop controller

Fig. 3. Effects of droop slopes and time constant in the LPFs in the frequency and voltage droop controllers applied in DQCI converter

As seen in Fig. 3 (a) and (b), the IPL limits can be affected significantly when changing the selected settings in both controllers, especially for settings in the voltage droop controller. It can be seen that with high droop slopes, i.e. from $70 \%$ to $300 \%$ and beyond, the IPL limits are almost not affected by changing the time constant in the LPFs, since the frequency or voltage response is quite small. The IPL limits in terms of voltage droop controller settings are generally lower than that of the frequency droop controller settings, with highest IPL limits at $92.7 \%$ and $86.1 \%$ respectively in this specific power system model. This indicates that the IPL limits appear to be more sensitive to changes in the voltage control and dynamics when compared with the frequency control and dynamics.

The results also suggest that, the aggressive droop slopes (in both frequency and voltage droop controllers) can have a positive effect on the IPL limits when the adjustment signal is properly filtered. For voltage droop controller, inadequate filtering on the droop adjustment can directly destabilise the system resulting in lower IPL limits. For the frequency droop controller, the highest IPL limits are achieved with a filter time constant $\tau_{L P F_{-} D f}$ of around $0.2 \mathrm{~s}$. It can be seen that allowing unfiltered frequency droop or excessive time delays/phase shifts (introduced by an inadequately tuned filter) potentially destabilises the system and thus lowers the IPL limit.

It should be noted that the IPL limits discussed in this paper are based on this simplified power system model and do not include the effects of the harmonics, inter-harmonics, unbalance, reactive load steps, and potentially more complex interactions between potentially millions of specific converters from different manufacturers with individual peculiarities. It is therefore, likely that the results are optimistic i.e. higher than those observed in a more detailed model or actual power system. Additionally, the viability of a particular 
configuration is highly dependent on the choice of many parameters (even in this aggregated model) including governors' time constants, droop slopes, network impedances and measurement time-windows. Changing just one parameter can sometimes radically alter the performance, and thus, the IPL limits. More detailed future studies could examine the effects of many more different factors, such as grid impedance and topology, load non-linearity, and their impact on the system stability limits.

Through the above investigations the IPL limit was maximised by selecting the optimised frequency and voltage droop controllers settings, i.e. droop slopes and time constants in the LPFs are selected using the optimised values of $D_{f}=60 \%, \tau_{L P F_{-} D f}=0.04 \mathrm{~s}, D_{v}=60 \%$ and $\tau_{L P F_{-} D f}=0.04 \sim 0.4 \mathrm{~s}$. The resulting IPL limits with the combined optimised settings are shown in Table 1, along with the original results. It can be seen that the IPL limit is improved with the combined settings. When the filter time constant is properly chosen for the voltage droop controller (in this case longer than $0.3 \mathrm{~s}$ ), a maximum IPL limit of $94.6 \%$ is achieved.

Therefore, in this case study, by combining the best droop controller options from the two independent investigations (i.e. frequency and voltage droop) the overall system stability limit has been enhanced. Such method can also be applied to other settings (or parameters) that have the potential to affect the IPL limit.

Table 1. Optimisation of IPL limits based on settings in both frequency and voltage droop controllers in the DQCI converter

\begin{tabular}{|c|c|c|c|c|c|c|}
\hline \multirow{3}{*}{\multicolumn{2}{|c|}{$\begin{array}{l}\text { IPL limits }(\%) \text { based on the } \\
\text { power system model }\end{array}$}} & & \multicolumn{4}{|c|}{ Voltage droop controller } \\
\hline & & \multirow{2}{*}{$\begin{array}{c}D_{v}= \\
\text { infinite }\end{array}$} & \multicolumn{4}{|c|}{$\tau_{L P F}(\mathrm{~s})$ with $D_{v}=60 \%$} \\
\hline & & & 0.4 & 0.3 & 0.2 & 0.1 \\
\hline \multirow{2}{*}{$\begin{array}{c}\text { Frequency } \\
\text { droop } \\
\text { controller }\end{array}$} & $D_{f}=$ infinite & & 86.1 & 86.1 & 86.1 & 86.1 \\
\hline & $\begin{array}{c}D_{f}=60 \% \\
\tau_{L P F}=0.04 \mathrm{~s}\end{array}$ & 92.7 & 94.6 & 93.3 & 90.2 & 84.8 \\
\hline
\end{tabular}

\subsection{Frequency response using NFP method}

As introduced in section 2.2, the response $R$ of a specific type of generation and its controllers can be analysed using the NFP method, either by classical analysis of the device's transfer functions or by timedomain simulation followed by Fourier Analysis. To carry out a classical analysis based on transfer functions, the main function blocks of the generation controllers have to be linear. However, it is difficult to construct a classical linearised model of the whole DQCI converter due to the dq-axis translations within the PLL and high-bandwidth inner current control loop, and the high number of non-linear blocks in the DQCI converter control system, such as ramp-rate limiters and multi-rate systems. In this section, the NFP results describing responses of the SG and DQCI converters will be presented based on the time-domain simulation analysis. The simulation model for NFP analysis has been validated by comparing the response of the SG obtained by both linear analysis and time-domain simulation, which have been found to be consistent with each other.

NFP charts of SG and DQCI converters are shown in (a) Amplitude chart

Phase chart 
Fig. 4 (a) and (b) for amplitude and phase respectively, marked as $R_{S G}$ and $R_{D Q C I}$, along with the three key features $R_{\text {Droop }}, R_{H}$ and $R_{H D}$, which were discussed earlier. Note that turbine and governor control has been modelled for the SG and their affect can be observed on the on the graph (see $R_{S G}$ ), while trend lines $R_{H}$ and $R_{H D}$ show the contributions from the inertia and $\mathrm{Xd}$ ' to $R_{S G}$. For a real synchronous machine attached to a prime mover, there is usually a band of modulation frequencies $f_{\text {NFPmod }}$ between these two initial regions for which the RSG response amplitude becomes quite small.

Note: Frequency droop slope is set to $4 \%$ and voltage droop controller is disabled for both SG and DQCI in this case shown.

The results indicate that the modulation frequency requires actions which are faster than the prime mover and governor are physically capable of, but that the modulation frequency is too slow to initiate a large inertial contribution. For a modulation frequency range of approximately 0.2 to $2 \mathrm{~Hz}$, the $\mathrm{SG}$ response $R_{S G}$ follows the inertia trendline $R_{H}$ (and $R_{H D}$ ) for both amplitude and phase, until it reaches a rotor oscillation mode at around $2 \mathrm{~Hz}$. For modulation frequencies higher than $2 \mathrm{~Hz}, R_{S G}$ (and $R_{H D}$ ) is dominated by the machine damping effect, where amplitude response decreases and phase lags with increasing frequency. The response of $R_{S G}$ follows $R_{H}$ and $R_{H D}$ in their corresponding modulation frequency regions as expected.

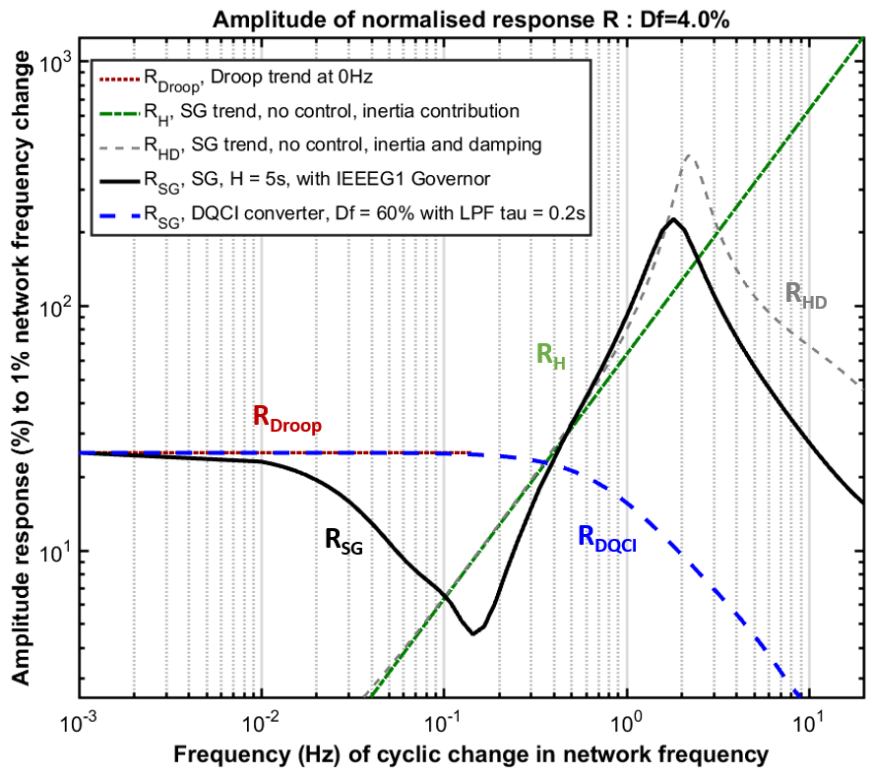

(a) Amplitude chart

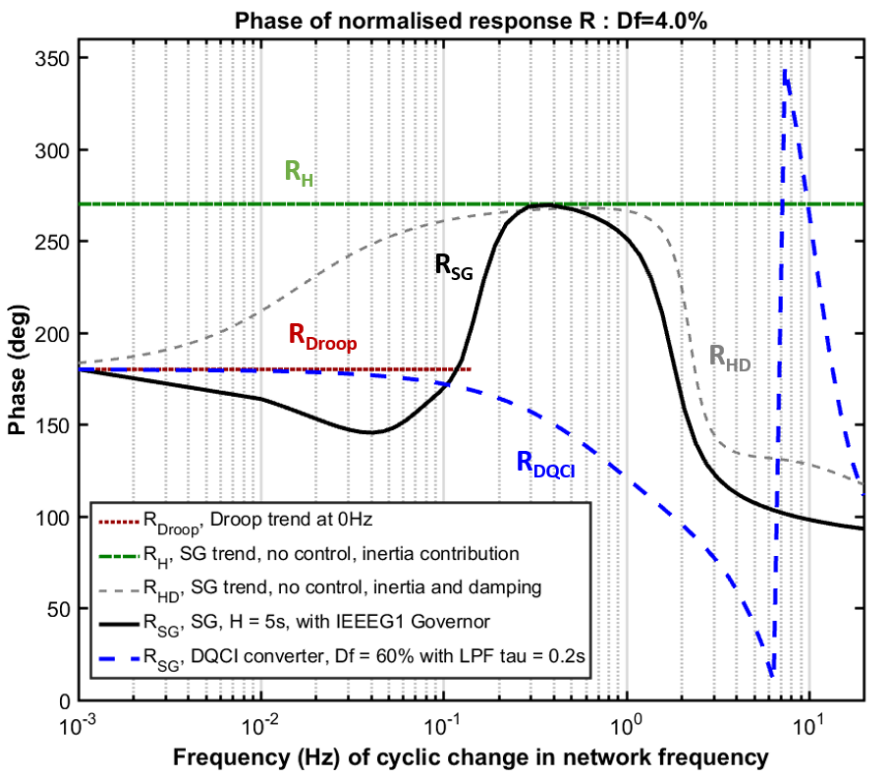

(b) Phase chart

Fig. 4. NFP charts in for SG $\left(R_{S G}\right)$ and DQCI converter $\left(R_{D Q C I}\right)$ obtained by numerical simulation along with the three key trend-lines $\boldsymbol{R}_{\text {Droop}}, \boldsymbol{R}_{H}$ and $\boldsymbol{R}_{H D}$ predicted by classical linear analysis

In comparison, the response $R_{D Q C I}$ is quite different. In the region $10^{-3}$ to $10^{-1} \mathrm{~Hz}$ it follows the droop asymptote according to the $4 \%$ frequency droop applied in the DQCI converter. It starts to drop off in amplitude for modulation frequencies above $0.2 \mathrm{~Hz}$, which does not follow the inertia trendline as expected. Similarly, the phase of $R_{D Q C I}$ begins to drop from around $0.02 \mathrm{~Hz}$ and experiences an even deeper slope after $4 \mathrm{~Hz}$. Note that the 'spike' seen in the phase chart of $R_{D Q C I}$ is the wrapped phase which is displayed in the range of 0 to 360 degree. This reduction in phase response of the DQCI converter can become anti-phase with the SG rotor oscillation. A significant phase difference up to $130^{\circ}$ between $R_{S G}$ and $R_{D Q C I}$ can be introduced 
when the SG is following the inertia trendline and even larger differences can be introduced at high modulation frequency regions beyond $4 \mathrm{~Hz}$, which can be easily affected by any settings in the DQCI control system and would be difficult to predict in any actual power system. Additionally, oscillations found in the power system model when approaching marginally unviable cases have a frequency in the range from $4 \mathrm{~Hz}$ to $8 \mathrm{~Hz}$, which is consistent with the NFP charts where massive phase differences are evident. Therefore, the steep phase slopes shown in the NFP charts can indicate a tendency for instability at high penetrations.

The highest / lowest IPL limit responses of DQCI converters with appropriate settings for the frequency droop controller (with the voltage droop controller disabled) are shown in (a) Amplitude chart (b) Phase chart

Fig. 5. Three cases have been selected corresponding to highest and lowest IPL limits achieved. For the worst case, the responses are similar to those shown in (a) Amplitude chart

\section{Phase chart}

Fig. 4. Conversely, for the best case, the amplitude response is generally smaller due to the high $D_{f}$ while the phase response has been improved significantly, i.e. less phase drop in $2 \sim 10 \mathrm{~Hz}$ range. This confirms that steep phase slopes can destabilise the system resulting in a lower IPL limit.

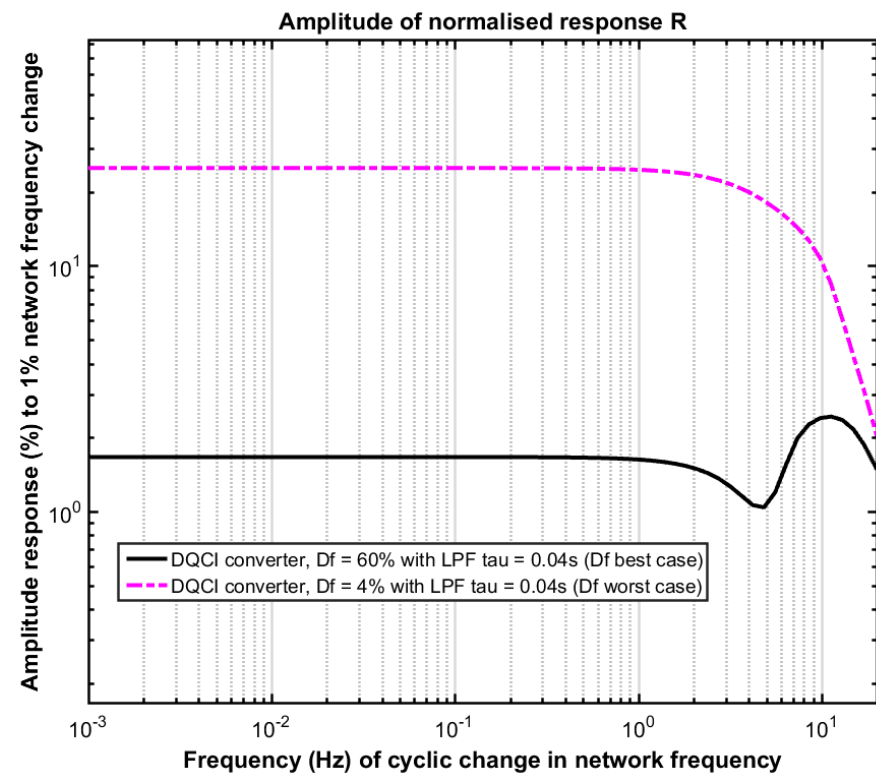

(a) Amplitude chart

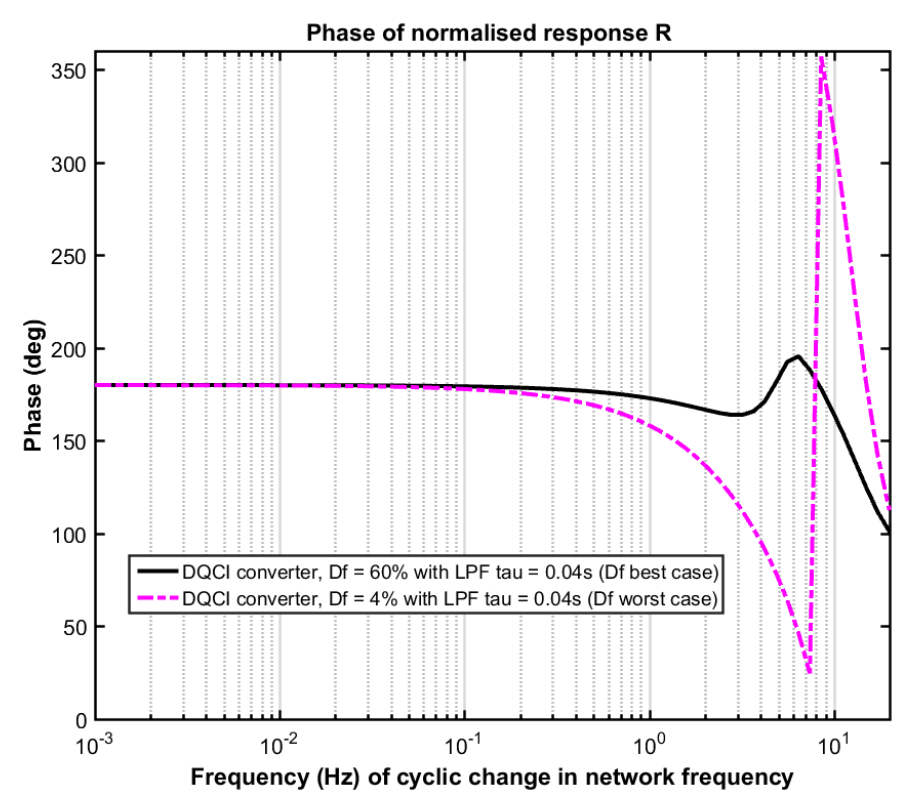

(b) Phase chart

Fig. 5. NFP charts for DQCI converter with different frequency controller droop slopes

From the above case studies, the NFP method is capable of analysing generation response to a change in network frequency and assisting in predicting system stability when various devices are interconnected and interact with each other. Further studies will be undertaken to fully analyse the information contained in the NFP charts. In particular, the NFP method can be utilised to analyse and help verify whether the device provides frequency support as expected, as has been investigated by the authors.

\section{Conclusions and future work}

With increased penetration of NSD, future power systems will be required to operate satisfactorily with much higher penetrations of converters. This paper has demonstrated and quantified the IPL limits (with 
respect to small disturbances) of the DQCI converters in a power system based on a set of assumed viability criteria. These limits are affected by frequency and voltage droop controllers applied in the DQCI control systems. It has also been demonstrated that there are various factors (in both power system simulations and in actual power systems) that could potentially affect the IPL limits, and it has been shown that the IPL limits can be maximised by combining the globally optimised controller settings. A method to analyse the responses of generation (both SG and NSD) to network frequency perturbations, i.e. the NFP method, has been introduced in this paper, which can be used to make a clear distinction between the devices of their frequency responses and provide visualisation analysis relating to the interaction of the devices when they are connected in the same network. The responses of SG and DQCI converters have been analysed using the NFP method in this paper and it has been shown that significant phase differences between the SG and the DQCI converters at certain frequency regions can potentially contribute to instability of the network at high penetration of converters.

It should be highlighted that the IPL limits demonstrated in this paper are based on a specific power system model and do not yet include the effects of harmonics, inter-harmonics, unbalance, or other phenomena that would be present in an actual network. Furthermore, the IPL limits will also clearly be influenced by the response of the system to other transients, such as fault events - and the limits influenced dictated by fault responses are expected to be lower than those specified using the NFP method. It is also likely that the IPL limits stated in this paper are higher than those that would be evident in either a more comprehensive power system model or in an actual power system. As the "viability" of a particular configuration in the model is highly dependent on the choice of many parameters and changing one parameter can sometimes radically alter the performance and IPL limits, the key value of the presented results lies in the observed trends of IPL limits and the analysis methodology rather than in their absolute values.

Future work should investigate effects of other system parameters that could potentially affect the IPL limits, such as DQCI controller gains, rate limitation on the converter power output, system impedance, etc. The IPL limit studies should be re-executed using a more comprehensive power system model and/or the converter hardware-in-loop (CHIL) environment to more realistically establish impact of those various factors on the IPL limits. Various types of converter controllers should also be tested in the future with the NFP method to ascertain their responses to network frequency perturbation, e.g. DQCI converters with different control settings, DQCI converters equipped with RoCoF frequency response provision, and other types of converter control algorithms such as virtual synchronous machine algorithms discussed in [23]. Finally, network voltage perturbation (NVP) method, which can be used as a companion of the NFP method, is presently under investigation by the authors to enable a study of the coupling between the frequency and voltage responses of power system, to further improve insight and understanding of the potential for instability in power systems with high penetrations of converters. 


\section{References}

[1] National Grid (UK), "Future Energy Scenario," 2015.

[2] National Grid (UK), "System Operability Framework," 2015.

[3] N. Ray Chaudhuri, B. Chaudhuri, R. Majumder, and A. Yazdani, "The Voltage-Sourced Converter (VSC)," in Multi-Terminal Direct-Current Grids, ISBN 9781118960486: John Wiley \& Sons, Inc, 2014, pp. 23-75.

[4] ENSTO-E, "ENTSO-E Network Code for Requirements for Grid Connection Applicable to all Generators," 2013.

[5] ENTSO-E, "ENTSO-E Network Code on High Voltage Direct Current Connections and DC-connected Power Park Modules," 2014.

[6] National Grid (UK), "The Grid Code," 2016.

[7] "IEEE Guide for Planning DC Links Terminating at AC Locations Having Low Short-Circuit Capacities," IEEE Std 1204-1997, pp. 1-216, 1997.

[8] L. Zhang, H. Lennart, and N. H. P., "Power-Synchronization Control of Grid-Connected VoltageSource Converters," IEEE Transactions on Power Systems, vol. 25, pp. 809-820, 2010.

[9] M. Durrant, H. Werner, and K. Abbott, "Model of a VSC HVDC terminal attached to a weak AC system," in Proceedings of IEEE Conference on Control Applications, 2003, pp. 178-182 vol.1.

[10] L. Zhang, "Modeling and Control of VSC-HVDC Links Connected to Weak AC Systems," School of Electrical Engineering (EES), Electrical Machines and Power Electronics, KTH, Stockholm, 2010.

[11] J. O'Sullivan, A. Rogers, D. Flynn, P. Smith, A. Mullane, et al., "Studying the Maximum Instantaneous Non-Synchronous Generation in an Island System: Frequency Stability Challenges in Ireland," IEEE Transactions on Power Systems, vol. 29, pp. 2943-2951, 2014.

[12] M. Nahid Al, Y. Ruifeng, T. K. Saha, and N. Modi, "Frequency response and its enhancement using synchronous condensers in presence of high wind penetration," in Power \& Energy Society General Meeting, 2015 IEEE, 2015, pp. 1-5.

[13] L. Meegahapola and T. Littler, "Characterisation of large disturbance rotor angle and voltage stability in interconnected power networks with distributed wind generation," IET Journal on Renewable Power Generation, vol. 9, pp. 272-283, 2015.

[14] M. Edrah, K. L. Lo, and O. Anaya-Lara, "Impacts of High Penetration of DFIG Wind Turbines on Rotor Angle Stability of Power Systems," IEEE Transactions on Sustainable Energy, vol. 6, pp. 759766, 2015.

[15] E. Vittal, M. O'Malley, and A. Keane, "A Steady-State Voltage Stability Analysis of Power Systems With High Penetrations of Wind," IEEE Transactions on Power Systems, vol. 25, pp. 433-442, 2010.

[16] H. R. Chamorro, M. Ghandhari, and R. Eriksson, "Influence of the increasing non-synchronous generation on Small Signal Stability," in PES General Meeting | Conference \& Exposition, 2014 IEEE, 2014, pp. 1-5.

[17] P. N. Papadopoulos and J. V. Milanovic, "Impact of penetration of non-synchronous generators on power system dynamics," in 2015 IEEE PowerTech, Eindhoven, 2015, pp. 1-6.

[18] H. Urdal, R. Ierna, Z. Jiebei, C. Ivanov, A. Dahresobh, et al., "System strength considerations in a converter dominated power system," IET Journal on Renewable Power Generation, vol. 9, pp. 10-17, 2015.

[19] M. Yu, A. J. Roscoe, C. D. Booth, A. Dysko, R. Ierna, et al., "Use of an Inertia-less Virtual Synchronous Machine to Stabilise Networks with High Penetrations of Converters," in Power Systems Computation Conference (PSCC), Genoa, Italy, 2016.

[20] IEEE Power \& Energy Society (PES), "Dynamic Models for Turbine-Governors in Power System Studies," 2013.

[21] SEM Committee, "SEM-13-098 DS3 System Services Technical Definitions Decision Paper," 2013.

[22] Ofgem, "Distribution Code G59," 2015.

[23] H. Bevrani, T. Ise, and Y. Miura, "Virtual synchronous generators: A survey and new perspectives," International Journal of Electrical Power \& Energy Systems, vol. 54, pp. 244-254, 2014. 